\title{
La evaluación de los movimientos repetidos en miembro superior El método OCRA
}

Juan Alberto Castillo M.

\section{Resumen}

El impacto de las lesiones asociadas a movimientos repetidos en miembro superior, en la economía colombiana y en la salud de los trabajadores colombianos es de tal magnitud, que varios de los síndromes asociados a este tipo de acciones se encuentran entre las cinco primeras causas de enfermedad profesional en Colombia, afectando principalmente a mujeres trabajadoras.

La morbilidad por desórdenes músculo-esqueléticos representa en Colombia el $43 \%$ de todos los diagnósticos de enfermedad profesional. (Minprotección, 2005). De acuerdo a datos de FASECOLDA, 2005 de 1493 enfermedades profesionales registradas, 393 involucran a miembro superior y ocasionaron más de 7000 días de incapacidad y pérdida de productividad.

Por las dificultades de identificación, diagnóstico, tratamiento y rehabilitación, este tipo de lesiones se han constituido en un reto para el sistema de seguridad social, el sistema de riesgos profesionales y para la diversidad de profesionales que tratan de desarrollar medidas para identificar, controlar y prevenir este tipo de lesiones.

En el presente se realiza un análisis de la complejidad que ofrece el estudio del miembro superior, luego las principales variables de análisis y finalmente se estudia el método OCRA (Occupational Repetitive Actions).

Palabras clave: OCRA, desórdenes músculo-esqueléticos, análisis del movimiento, análisis de tareas.

\section{Evaluation of repeated movements in superior limbs, the OCRA method}

\section{Abstract}

The impact on the Colombian economy because of the injuries associated to the repetitive movements in the Upper Limb in the health of the Colombian workers has such a magnitude that many of these

\footnotetext{
Diseñador industrial, Magíster Ergonomía, PhD Grupo de Investigación GiSCYT. Universidad del Rosario. Juan.castilloma@urosario.edu.co
} 
syndromes (associated with this type of tasks), are located among the first five causes of occupational disease in Colombia, affecting manly women.

The morbidity for muscle skeletal disorders represent in Colombia $43 \%$ of all the diagnoses of occupational disease. (Minproteccion, 2005). In agreement to information of FASECOLDA, 2005 of 1493 registered occupational diseases, 393 involve the Upper Limbs and they caused more of 7000 days per year of disability and loss of productivity.

For the difficulties of identification, diagnose, treatment and rehabilitation, this type of injuries have been constituted a challenge for the security social system, the risks labor system and for the others professionals.

Therefore this paper show an analysis of the complexity that offers the study of the Upper Limbs, after the principal variables of analysis and finally there is studied the method OCRA (Occupational Repetitive Actions).

Key words: OCRA, muscle skeletal disorders, movement analysis, tasks analysis.

El estudio de las lesiones osteomusculares asociadas al trabajo reviste gran complejidad, por el número de variables implicadas y por las dificultades para apreciar la función y peso de cada una de éstas en el desencadenamiento de una lesión. Es complejo por las dificultades que significan en el estudio de una actividad, el aislar la actividad de trabajo sin afectar su estructura. Esto limita el análisis y hace que sea más compleja la definición de unidades de análisis que den cuenta de forma integral del fenómeno de las lesiones osteomusculares en la dinámica particular de un trabajo.

En consecuencia al estudiar las lesiones osteomusculares, parece difícil imaginar el análisis de los movimientos asociados a estas patologías sin considerar la existencia de un referencial. Todo movimiento está orientado y se relaciona con objetivos físicos, espaciales y cognitivos. En el desarrollo de un movimiento en el trabajo la selección de trayectorias espaciales o la disposición del individuo en el espacio está relacionada directamente a un referente, por tanto, no es posible pensar que sea posible efectuar el menor movimiento sin que se disponga de un referente, sea este cognitivo (la belleza del movimiento mismo, por ejemplo) o físico (maximizar la eficiencia mecánica del esfuerzo realizado). Estos elementos resultan más complejos cuando se trata del estudio de los movimientos asociados a actividades que involucran los (MMSS)Miembros Superiores. Este trabajo aborda entonces dicha problemática, evaluando al tiempo la pertinencia de un método específico de estudio del movimiento repetido en miembro superior.

\section{Complejidad del movimiento en miem- bro superior}

El impacto de las lesiones asociadas a movimientos repetidos en miembro superior en la economía colombiana y en la salud de los trabajadores colombianos; es de tal magnitud que varios de los síndromes asociados a este tipo de acciones se encuentran entre las cinco primeras causas de enfermedad profesional en Colombia, afectando principalmente a mujeres trabajadoras.

La morbilidad por desórdenes músculoesqueléticos representa en Colombia el $43 \%$ de todos los diagnósticos de enfermedad profesional. (Minprotección, 2005). De acuerdo a datos de FASECOLDA, 2005 de 1493 enfermedades pro- 
fesionales registradas, 393 involucran a miembro superior y ocasionaron más de 7000 días de incapacidad y pérdida de productividad.

En este sentido se puede decir que las tareas productivas que involucran movimientos repetitivos en miembros superiores, pueden desencadenar alteraciones osteomusculares y del sistema nervioso que llegan a comprometer hombro, codo, mano, muñeca debido a la realización de actividades manuales bajo patrones específicos y estereotipados por largos periodos de tiempo.

$\mathrm{Al}$ abordar esta problemática, diversos problemas se hacen presentes en el desarrollo de estos estudios. En primer lugar, los problemas de modelamiento matemático de las trayectorias espaciales, en segundo lugar la redundancia de datos requerida para establecer patrones de referencia y en última instancia la influencia del contexto de acción y de las características de los individuos.

Adicionalmente en el estudio del movimiento humano en general y de los miembros superiores en específico, es de difícil predicción el uso de estrategias desarrolladas por los individuos, por que modifican en gran medida patrones y trayectorias de los elementos de referencia seleccionados; para el estudio del movimiento (articulaciones, segmentos corporales, estructuras óseas).

A este respecto hay que decir que el estudio de la cinemática de las extremidades superiores, obedece a movimientos que reúnen una doble complejidad, de un lado son movimientos rápidos que al tiempo describen múltiples trayectorias espaciales lo que hace delicado el estudio tridimensional del movimiento. De otro lado las articulaciones del miembro superior, conjugan una diversidad de posibles movimientos, lo que otorga gran flexibilidad al miembro superior, pero que al tiempo hace complejo su estudio debido a los grados de libertad.

Tomando como ejemplo, el complejo articular del hombro, se ve que está constituido por cinco articulaciones, la complejidad organizacional y funcional de estas cinco articulaciones hace delicado el análisis de trayectorias y definición precisa de la posición del miembro superior, principalmente por la flexibilidad de la misma y los grados de libertad que proporciona (ver figura 1).

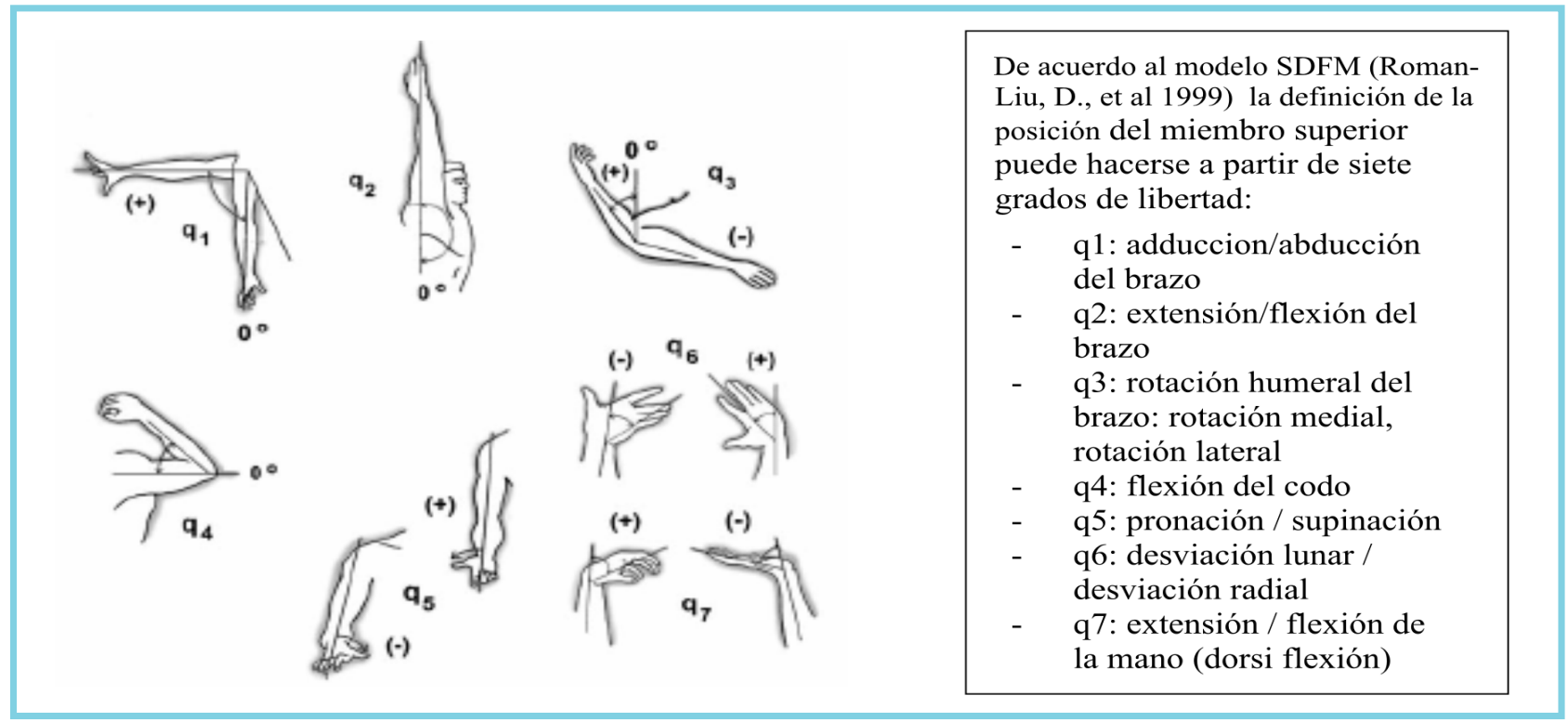

Figura 1. Determinación de la postura del miembro superior a partir de la definición de los grados de libertad de acuerdo a Roman-Liu, D. 1999. 
Se tiene en cuenta que los movimientos y transiciones de movimiento del hombro involucran tanto a huesos como a músculos, debe integrar el análisis de la posición relativa del tronco y su influencia en la posición, velocidad y aceleración de los movimientos desarrollados por los miembros superiores, lo cual hace necesario un estudio cuidadoso de las posibles combinaciones de estos elementos en la producción del movimiento.

\section{Los movimientos repetidos}

En la mayoría de los estudios relativos a los movimientos repetidos se busca establecer índices que permitan a través de diferentes medios y métodos identificar los posibles riesgos de lesión o los posibles precursores de estas lesiones. Entre las diversas estrategias empleadas se encuentran los estudios que basan su búsqueda en el uso de la electromiografía de superficie de músculos específicos (Jones and Hunter, 1983; Cook et al., 1998; Mathiassen, 1993; West et al., 1995; Bystrom and Kilbom, 1990; Roman-Liu et al., 2004). Otros han sido desarrollados con el fin de identificar la percepción individual de los sujetos expuestos a las condiciones de repetitividad establecida (Mathiassen, 1993). Otros han enfocado su trabajo en la identificación de los aspectos biomecánicos relacionados con el estudio de la carga y sus efectos en las estructuras músculo-esqueléticas, asociando los aspectos relativos al diseño y a la dinámica del proceso de trabajo (Cook et al., 1998; Bystrom and Kilbom, 1990; Dahalan and Fernández, 1993; Mathiassen, 1993; Hagberg, 1981; Roman-Liu et al., 2005; Bao et al., 2006a, b) De manera general se puede decir que estos estudios buscan establecer relaciones entre las características y exigencias de las tareas con los efectos de éstas en términos de carga física y de efectos en el sistema osteomuscular.

De estos estudios se concluye que para llevar a cabo correctamente el estudio de los movimientos repetidos de miembro superior en tareas industriales, es necesario tomar en cuenta que la repetición de una acción está directamente vinculada al logro de un objetivo de producción, esto significa que el diseño de la secuencia de acciones distribuidas entre la máquina y el hombre, predefinen la velocidad, frecuencia, esfuerzo e intensidad de la acción que un individuo deberá realizar.

Por tanto, la intervención de un individuo en un proceso, estará directamente relacionada con el diseño de la tarea. Por lo que es importante considerar en primer lugar los aspectos técnicos y organizacionales del proceso que se desea analizar, también es necesario establecer que las magnitudes de medida y de referencia en el estudio de la repetición son el tiempo asignado, la precisión de la acción y la fuerza requerida.

Desde el punto de vista de la repetición se debe considerar no sólo la interacción, la cual puede ser definida como las acciones y retroacciones que permiten al individuo y a la máquina obtener un resultado. También es necesario considerar las iteraciones que se producen por fallas operacionales o funcionales y que son concebidas y puestas en funcionamiento por el individuo de acuerdo a la naturaleza del contexto de acción, éstas últimas pueden ser estudiadas a partir del número de veces (frecuencia) que se debe ejecutar una misma acción. De otro lado estas iteraciones pueden ser de dos órdenes: las que están asociadas al diseño de la tarea y las que obedecen a disfuncionamientos técnicos $\mathrm{u}$ organizacionales.

La importancia de reflexionar separadamente estas dos variables de análisis reside en que las interacciones son fácilmente identificables, es posible repertoriarlas e inclusive calcular su frecuencia, esto en razón a que son parte constitutiva del diseño de la tarea y pueden ser registradas por un observador externo. Las iteraciones, son más difíciles de identificar ya que su aparición se asocia a la presencia de un evento perturbador, lo que hace que cada individuo disponga o desarrolle estrategias que le permiten encontrar una solución, éstas se asocian a periodos de funcionamiento en los cuales los equipos con los que se interactúa sufren problemas técnicos y deben ser ajustados. De igual forma resultan de las modificaciones no previstas 
de programas de producción, las cuales obligan en muchos casos a la adopción de estrategias específicas de operación. Por tanto, son particulares a los individuos, y no es posible o es difícil identificarlas por un observador externo sin la explicación del individuo ejecutante.

Ese puede entonces decir que estas acciones incluyen estrategias de intervención sobre componentes o elementos bien definidos. Para un observador, estas estrategias sólo podrán ser identificadas cuando el evento se produzca, es decir, es necesario establecer previamente los tipos de eventos que se producen en el desarrollo de la tarea. La identificación de este tipo de acciones parece relevante para el estudio de las lesiones osteomusculares de miembro superior, por las exigencias en cuanto a velocidad de respuesta (ejecución de movimientos rápidos) y por las demandas de precisión (preparación ext ante de la acción).

\section{El índice OCRA}

El índice OCRA, (Colombini, 1998 y Ochipinti et al., 2000, Colombini et al., 2002), busca establecer criterios, procedimientos y herramientas para estimar y evaluar la exposición a lesiones osteomusculares en miembro superior. El índice OCRA proporciona información basada en la exposición colectiva y datos de salud referidos a la manifestación gradual en mano y en el tiempo.

Estos datos proveen valores de referencia preliminar para clasificar los resultados en tres áreas: riesgo ausente, incierto o riesgo bajo y riesgo presente. Este es un modelo predictivo que emplea ecuaciones de regresión linear simple para estimar la prevalencia esperada (expresada por la variable Prevalencia individual casos de diagnóstico clínico de lesiones osteomusculares de miembro superior por 100 sujetos expuestos).

El índice se obtiene al establecer la relación entre el número actual de acciones técnicas ejecutadas durante un turno de trabajo (ATA) y el nú- mero de acciones técnicas de referencia (RTA) (para cada miembro superior), el cual se determina de manera específica de acuerdo al escenario de trabajo.

El proceso de evaluación significa tomar en cuenta los tres siguientes pasos:

1. Cálculo de la frecuencia de las Acciones Técnicas por minuto (AT/min.) y de manera global en número Actual de Acciones Técnicas (ATA) llevadas a cabo en un turno (para cada miembro superior).

2. Se calcula el número global de Acciones Técnicas de Referencia dentro de un turno (RTA).

3. Cálculo del Índice OCRA y evaluación del riesgo.

La identificación de acciones técnicas, implica que se debe hacer una caracterización de la repetición, con el fin de determinar cuáles tareas serán objeto de una evaluación. Esta caracterización implica que toda tarea repetitiva para el miembro superior, debe analizarse si su repetición implica la ejecución consecutiva por al menos 1-2 horas al día, con ciclos de trabajo similares entre sí y con una corta duración (algunos minutos).

Una vez se establece cuáles tareas son actualmente repetitivas y que pueden ser sometidas a un análisis, el problema más importante es la cuantificación/evaluación de la repetición. Para establecer la frecuencia, se recomienda contar de manera cuidadosa y analítica todas las acciones técnicas definidas en el método mover, alcanzar, tomar, tomar con una mano, tomar de nuevo con la otra mano, posicionar: insertar, extraer; presionar, halar, liberar; presionar inicio con un botón transportar, considerar también las acciones específicas a cada proceso, por ejemplo cepillar, girar, doblar, recortar, estrujar, ajustar, armar, dar formar, configurar; compactar, reducir; batir, golpear; frotar, pulir, pintar; rayar; limpiar, pulir, martillar, arrojar, lanzar. 
Cada una de estas acciones debe ser descrita y contada de manera individual para cada repetición, por ejemplo: girar dos veces al cerrar una llave, se registra como dos acciones técnicas.

De manera simultánea se debe efectuar un análisis de posturas de tipo cuantitativo y cualitativo, este análisis debe diferenciar, así como considerar los componentes estáticos y dinámicos del movimiento. Esto debe permitir obtener una estimación general del grado de repetición y la duración del movimiento en cada articulación dentro de cada secuencia de acciones técnicas.

La descripción analítica de las posturas puede desarrollarse considerando para el análisis, las acciones técnicas que requieren posturas y movimientos de cada segmento que van más allá de los niveles críticos de excursión angular. Acciones técnicas que involucran posturas o movimientos estáticos con aceptable excursión angular pero que deben mantenerse o repetirse de la misma manera y la duración, expresada en fracciones de ciclo/ tiempo de tarea. De cada una de las condiciones reportadas anteriormente. Esta combinación de factores, debe permitir la elaboración de una clasificación del esfuerzo para cada segmento considerado.

Para esto se realiza descripción de las posturas o movimiento, se considera de manera separada cada miembro superior. Se establece el área de riesgo para cada articulación considerada postura o movimiento no confortable esto se lleva cabo con referencia al porcentaje de tiempo del ciclo en el cual el movimiento o postura está presente. Esto permite establecer el multiplicador de postura (POm), establecer la repetición de ciertos movimientos, considerar el multiplicador de repetición (Rem).

Seguidamente se establecen los periodos de recuperación, aquí se consideran los periodos asignados para refrigerios y alimentación, estos periodos se establecen mediante observación por varias horas, no se debe tener en cuenta sólo los periodos teóricamente asignados, ya que al realizar la observación de las acciones técnicas repetidas se puede encontrar que no se dispone de adecuados periodos de recuperación. Los periodos de recuperación son los que se producen en los periodos de trabajo antes del almuerzo y después de éste cuando se realiza jornada de trabajo dividida en dos periodos iguales de cuatro horas. Cuando se trabaja en periodos continuos de ocho horas, se debe identificar el periodo de desayuno, almuerzo o comida y se observan los ciclos de tiempo antes y después de este evento, luego se establecen los periodos de recuperación al interior de estos ciclos.

Acto seguido se establece el multiplicador de duración (Dum), éste da cuenta de la presencia de las tareas repetitivas en un periodo relevante del turno de trabajo, aquí se debe tomar en cuenta que en algunos escenarios de trabajo el trabajo repetitivo sólo se realiza en una parte del turno o en la mitad del tiempo, etc.

Finalmente se establece el Índice OCRA, comparando para cada miembro superior el número actual de acciones técnicas realizadas en el periodo de trabajo y la referencia del número de acciones técnicas en un turno. Se obtienen los valores críticos, siguiendo un código de colores: verde no Hay Riesgo, amarillo: Riesgo Bajo y rojo Riesgo Presente. Al tiempo se hacen sugerencias de acuerdo a las consecuencias: Verde: aceptable), Amarillo: medidas organizacionales o mejoramientos relativos a postura, fuerza, acciones técnicas, Rojo: Rediseño de las tareas y expuesto de trabajo de acuerdo a las prioridades recomendadas.

Se debe considerar que el índice OCRA sólo es útil para establecer la presencia de riesgo; sin embargo, la precisión en la estimación depende de una observación cuidadosa, del registro en video de las acciones, de un análisis cuidadoso de la fuerza ejercida, de los periodos de recuperación, entre otras variables por estas razones en aras de 
obtener confiabilidad, su aplicación debe ser cuidadosa y sólo deberá ser aplicada por especialistas entrenados no sólo en el método, sino principalmente en el estudio del trabajo.

\section{Materiales y métodos}

Con el objetivo de evaluar la aplicación del método OCRA, se realiza el estudio de movimientos repetidos en la actividad de trabajo de $10(n=10)$ agentes de servicio en la unidad administrativa de gestión de información y registro de datos de una empresa de servicios. Para cumplir con el objetivo se realiza el estudio en paralelo de las actividades de trabajo y se aplica el cuestionario simplificado OCRA.

Modelo de análisis de la situación de trabajo

El análisis de situaciones de servicio reposa sobre una mirada crítica que busca determinar "quién" produce y "qué" es lo que se produce en una situación de servicio, se trata de entender cómo evaluar lo que es producido.

El modelo de análisis empleado, está centrado en el estudio de la interacción de los diferentes actores, el análisis de la situación se fundamenta en dos nociones bastante cercanas como son las de Cooperación y la de Actividad Colectiva. Para este estudio se considera la cooperación como una " $a c$ tividad de gestión de interferencias producto de las actividades distribuidas entre diferentes agentes" . Considerando que la Actividad Colectiva se fundamenta en la elaboración y mantenimiento de un referencial $^{2}$ común que juega en lo colectivo el mismo rol de la representación ${ }^{3}$ individual”.

En el estudio de situaciones de servicio en las cuales se estudia un prestatario del servicio y un beneficiario del servicio, se prefiere hablar de agente. En razón a que la actividad central se orienta al agenciamiento de los intereses del beneficiario en función de los objetivos del prestatario".

2 Se refiere a un conjunto de principios y reglas para actuar en una situación dada.

Se refiere al proceso de elaboración de modelos de cada situación, que permiten el uso y desarrollo de estrategias y procedimientos de trabajo.
Desde el punto de vista metodológico el estudio de las comunicaciones y de gestión de la Información. Se han estudiado a través de: documentación, entrevistas individuales y colectivas, reuniones y observación directa de las prácticas de trabajo. Se han explorado igualmente las comunicaciones que se desarrollan en el curso de la actividad del agente de subsidios; éstos se han recolectado por observación y registros de actividad, identificando las formas de cooperación, de coordinación en el seno de los colectivos y en los aspectos operatorios del trabajo.

Los momentos de comunicación más autónomos - registro de solicitudes, relaciones con el cliente y con otros actores de la situación de servicio- han sido de igual manera observados y registrados. Éstos completan la Información y permiten acceder a las exigencias y estructura de la situación de trabajo.

El exámen de estos datos hacen aparecer los procesos de construcción y significación de la acción. Así el estudio ergonomía desarrollado busca estructurar un modelo tipo por reagrupación de las características de la actividad a partir de los comportamientos tipo (aspectos fisiológicos, por ejemplo), de situaciones tipo (sesiones de Interacción) y de lenguajes de descripción tipo (análisis en términos de elementos de significación).

El análisis de la situación de trabajo se lleva a cabo en una unidad especializada de la organización estudiada. Se trata de trabajadores que centran su actividad en la prestación de un servicio que responde a la estructura de la empresa. La problemática de la situación de trabajo se refiere al "conjunto coherente de criterios que permiten establecer las condiciones en las cuales se lleva a cabo un trabajo" éstos son:

- Contenido del trabajo: naturaleza de las tareas, subespecialización de tareas y diversidad de servicios. 
- Entorno de trabajo: características físicas de trabajo, distribución espacial, recursos en mobiliario y en dispositivos informáticos.

- Organización del trabajo: ciclos de trabajo, horarios, rotación de puestos de trabajo, funciones.

- El gasto físico y mental, asociado a las exigencias del servicio y a la naturaleza de la tarea misma.

El trabajo estudiado se desarrolla de acuerdo a los ciclos especificados, esto significa que en el transcurso del mes y de la semana las demandas de servicio tendrán intensidades diferentes. El número de clientes a atender, la complejidad de las solicitudes de servicio y los tiempos de atención por beneficiario, se encuentran vinculados a la calidad de la información disponible, así como a las verificaciones de datos que se requieren.

Este desarrollo en ciclos de intensificación de las demandas de servicios, lo que afecta la posibilidad de organizar la estructura del trabajo lo cual reduce y/o condiciona a la estrategia de cooperación establecida por el grupo de agentes de servicio. Es decir, quienes prestan directamente el servicio y mantienen el contacto con el beneficiario, así como con los agentes de soporte. Este aspecto central en el estudio de la situación establece las características medulares del trabajo, esto es:

a. una situación de trabajo por ciclos de demanda de servicio con:

- periodos de intensificación de demandas del servicio específico

- periodos de intensificación de verificación y control de información

- periodos de intensificación de las actividades de registro de datos

- periodos de actividad diferenciada por cargo

b. una situación con demanda de competencias múltiples para
- verificación de legitimidad de datos y validación de datos

- gestión de solicitudes con información de complejidad diversa

- identificar, validar e informar anomalías

- gestionar situaciones de agresión física y/o verbal

c. una situación caracterizada por acciones de

- acompañamiento y asistencia específica

- cooperación y coordinación

- interacción interna y externa

Estos tres elementos permiten establecer que la situación de trabajo debe ser estudiada como el resultado de conjunto de acciones que se conjugan para determinar la duración, complejidad, grado de exigencia física y cognitiva para cada uno de los cargos analizados.

\section{El servicio en la unidad analizada}

Como se aprecia, los agentes de la unidad de servicio centran su trabajo en la gestión y verificación en continuo de datos ${ }^{4}$. Estos datos permiten controlar la veracidad de las informaciones y la pertinencia de los pagos. Esto se hace en cada uno de los estados del proceso: desde la recepción de documentos, análisis de informaciones registradas, equivalencias encontradas y determinación de beneficios. Se puede entonces agrupar las tareas del departamento de servicios a partir de las siguientes operaciones:

a. recepción y registro de solicitudes

- empresarial

- personal

b. tratamiento y verificación de las solicitudes

c. cálculo y registro

d. generación de los registros

4 La noción de dato hace referencia a informaciones articuladas a finalidades, en este caso determinar la naturaleza del beneficio. 
e. pagos y verificación

f. rectificaciones y ajustes de datos

g. verificaciones de transacciones

h. control integral del proceso

La unidad de servicios exige a los agentes que la componen, un trabajo intensivo de tratamiento de documentos e informaciones que deben ser analizados, contrastados y verificados con el fin de estructurar los datos que permiten definir el cálculo de los beneficios. Este proceso requiere que los agentes de servicio hagan uso continuo de la atención, ya que en cada una de las etapas se debe evitar la producción de errores por la magnitud del costo financiero, de igual manera las inconsistencias en el registro y manejo de la información puede significar pérdidas en los contenidos de los datos y consecuentemente la necesidad de re-procesos.

Esto significa que esta unidad se ordena en tres grandes tipos de actividades:

\section{I. recepción y codificación de documentos: se} caracteriza por un intercambio de información entre el agente de servicio y el beneficiario, el objetivo es establecer la pertinencia de las informaciones suministradas. La mayoría de las interacciones se da entre el agente y el beneficiario, algunas interacciones se centran en la verificación de validez de informaciones entre los agentes de servicios.

II. gestión y registro de información: se caracteriza por un intercambio de información entre los agentes de servicio y los agentes de backoffice. Se trata de una gestión interna de la información con el objetivo de darle estructura a los datos que permiten posteriormente prestar el servicio en un marco de seguridad en cuanto a la calidad de éstos.

III. análisis y validación de los datos: se constituye en el elemento neurálgico del servicio, los datos son analizados cruzando los intereses del demandante del servicio con los intereses y ob- jetivos de la caja de compensación. Los agentes deben proceder a procesos de verificación interna de la validez de los datos y a proceso de verificación externa con los demandantes del servicio.

Se puede entonces decir que el servicio de subsidios ordena y organiza sus acciones en función de las informaciones tratadas, esto con el objetivo de estructurar datos que les den la posibilidad de prestar un servicio adecuado a los solicitantes. De esta manera el ingreso de información y la entrega del subsidio se constituyen en los extremos de secuencia.

Esto significa que a lo largo del proceso los agentes interactúan entre sí con el objetivo de certificar la veracidad de los datos y de esta manera asegurar que el manejo pertinente de los datos certifica que el pago corresponde a los requerimientos.

En el caso de esta unidad de servicio, se debe estudiar en doble dimensión: de un lado como una actividad que se articula a partir de una adecuada gestión de datos, de otro lado como una actividad de verificación de validez de estos datos entre agentes y esencialmente con los demandantes del servicio. En este último aspecto se debe considerar que el proceso de verificación requiere espacios de privacidad, ésta se ve afectada por las características físicas del espacio (diseño del área).

\section{Una relación de servicio especializada}

Esta relación de servicio puede ser definida como el conjunto de interacciones (relaciones entre actores humanos, tal que las acciones de unos influye o busca influir sobre el comportamiento de otros) que se desarrollarán entre agentes prestatarios (representante de la organización) y agentes utilizadores (clientes) a propósito de la prestación de un servicio.

El eje de la relación de servicio es el proceso que va de la solicitud a la respuesta, el mismo define los roles y funciones de los agentes. Estos roles 
implican la especialización (registro y orientación) y subespecialización de tareas (reprocesamiento de documentos, verificación de datos). Los agentes pueden ejecutar tareas especializadas y además poseer las competencias para algunas tareas subespecializadas. El trabajo en la unidad de servicio se encuentra organizado en función de dos grandes ciclos: verificación, cálculo, de otro lado orden de entrega y atención de reclamaciones. Estos dos ciclos definen la concertación de actividades en dos grandes periodos de tiempo del mes. Las actividades desarrolladas en la unidad se describen a partir de la función principal que las caracteriza, a continuación una descripción de ésas:

\section{El agente de tratamiento de datos}

Se constituye en una sección especializada de la unidad de servicios, se ocupa de la trascripción de documentos, de la verificación de informaciones y del registro de datos. Esta función se realiza con el tratamiento de los documentos en lotes (5080 formularios) que son registrados, verificados y validados en cuanto a calidad y veracidad de las informaciones suministradas.

El tratamiento de los lotes de documento puede ser asumido como una tarea subespecializada por agentes de pago o por agentes de registro de información. Es una tarea que implica velocidad de tratamiento y exactitud de registro en la plataforma de software que estructura los datos y posibilita la verificación de la vigencia de las informaciones y permite el cálculo de los derechos.

\section{El agente de Back-Office}

Se constituyen en el soporte para el desarrollo de las demás funciones de los agentes, se ocupan de la gestión, administración y control de las informaciones que les permiten proveer los cálculos. Se constituye en la sección que regula la operación y funcionamiento de la unidad desde el punto de vista de tratamiento y producción de datos estructurados y confiables.
$\mathrm{Su}$ función principal se encuentra articulada a la gestión de la información registrada en la plataforma tecnológica. Al control de la información proveniente de las empresas y de los usuarios individuales. A la consolidación de datos, el agrupamiento y categorización de las informaciones.

\section{Resultados del cuestionario OCRA}

La identificación de las acciones técnicas básicas permite establecer que las agentes de servicios desarrollan su trabajo en dos ciclos de cuatro horas con pausas de 10 minutos y una pausa de dos horas al medio día. Sin embargo, en picos de alta demanda del servicio, las pausas desaparecen y el servicio prolonga el tiempo de servicio de manera continua.

El trabajo desarrollado por las agentes se compone de verificación de datos, registro de datos utilizando teclado y raton, digitación y búsqueda en base de datos y consultas con los agentes próximos. Esto significa realizar movimientos repetidos de digitación; sin embargo, éstos se presentan en ciclos de 60 a 180 segundos de manera continua, con periodos de recuperación entre consultas. En picos de alta demanda el ciclo se reduce, pero se intensifica el número de usuarios lo que significa mayor continuidad en la ejecución de tareas.

El ciclo de trabajo se desarrolla entonces de esta manera. Cada agente debe procesar solicitudes consignadas en formularios (grupos de 50-80), cada formulario contiene en promedio 20 datos (160 caracteres en promedio). Esto significa repetir la acción técnica de golpear la tecla de manera continua a razón de entre 8000 y 12800 repeticiones por lote de formularios. Las agentes presentan rendimientos variados, esto es algunas de ellas realizan $150 \%$ de la cuota establecida y otras alcanzan sólo el $70 \%$ de ésta. Esto significa que algunas de las agentes procesan 120 formularios por jornada de trabajo, aproximadamente 19000 golpes de tecla. 
El trabajo se desarrolla en posición sedente a lo largo de toda la jornada, las agentes utilizan el tercio medio y el tercio anterior de la silla, emplean soportes lumbares adaptados (cojines), para mantener la postura. Algunas de ellas digitan utilizando soportes para documentos delante de la pantalla, otras utilizan soportes laterales y algunas ubican los formularios en las piernas. Esto implica el trabajo contiguo con cabeza y cuello en flexionados, el tronco en leve flexión y torsiones de 30 a 45 grados. Se presenta flexo extensión del codo, con desviación radial y flexión de muñeca, lo que significa mantener la posición de la muñeca por más de $1 / 3$ del tiempo de trabajo.

Este desarrollo no requiere el uso de fuerza extrema; sin embargo, se requiere mantener la postura y se producen gestos estereotipados de digitación de manera continua a lo largo de la jornada de trabajo. Se presentan movimientos de manos rápidos con cortas interrupciones.

\section{Conclusiones}

En el sentido estricto, la economía de los servicios recubre todos los sectores en los cuales una organización suministra un servicio a un particular, un grupo o una organización. En el caso de la organización analizada se puede decir que ésta es prestataria de servicios para individuos y para otras organizaciones, el punto de conexión entre estas dos organizaciones es el agente de servicios.

El interés esencial de un análisis de estas actividades es el de establecer las complejidades y dificultades propias de la interacción que se presenta entre el prestatario del servicio - agente- y el usuario o beneficiario del servicio. El puente de intercambio y de traducción de las necesidades del prestatario y del beneficiario del servicio es el "Agente" (asistente administrativo) de la organización prestataria. Esta situación presenta diferentes contextos de acción para el agente, dichos contextos varían en relación al tipo de tarea asignada al agente, a las metas fijadas por la organiza- ción prestataria y a las necesidades de la organización beneficiaria.

El agente de servicio se encuentra situado en un cruce de informaciones que son de vital importancia para gestionar la calidad del servicio, cumpliendo con las metas individuales y de grupo, éstas últimas poseen un valor esencial ya que a pesar de actuar individualmente en la mediación e intercambio de las organizaciones, el agente debe también actuar cooperativamente con los demás agentes de la unidad de servicio para alcanzar objetivos comunes. Es por tanto necesario tomar en cuenta que estos objetivos son uno de los indicadores de eficiencia colectiva y juega un rol esencial en la armonía operacional del grupo.

Adicionalmente, las condiciones de trabajo de los agentes se encuentran co-determinadas por las condiciones que le plantean las dos organizaciones a las que el agente atiende. Las relaciones existentes y que se generen entre las dos organizaciones influyen el trabajo desarrollado por el agente, ya que un número relativo de tareas que el agente debe desarrollar en ocasiones son tomadas a cargo por actores de la división de apoyo (Back office operations ${ }^{5}$ ), que no tienen contacto directo con el cliente y que si no son desarrolladas adecuadamente dificultan la actividad que el agente lleva a cabo, generando situaciones de incomprensión en la relación de interacción del agente con el beneficiario del servicio.

Las actividades de servicio se pueden caracterizar en tres categorías de acuerdo a la naturaleza del servicio (que se ofrece) y a la función que cumple el prestatario,

- Situaciones de servicio en las cuales la oferta de servicio está preconcebida y el beneficiario

Se refiere a las operaciones de soporte que deben desarrollarse para llevar a cabo con éxito la prestación del servicio, de alguna manera son el conjunto de operaciones que condicionan la calidad del servicio prestado. 
deberá seleccionar en un abanico de opciones preestablecidas.

- Situaciones de servicio en las cuales la oferta se adapta a la solicitud específica del usuario, de acuerdo a unos márgenes de ajuste limitado.

- Las situaciones en las cuales el servicio se diseña a la medida de las necesidades específicas del usuario (asistencia y consejo para resolver un problema específico).

En las actividades de servicio, los aspectos organizacionales adquieren un carácter central. El trabajador (agente) que desarrolla su trabajo en interacción con clientes necesita diversos recursos técnicos y operativos que regularmente provienen de las actividades llamadas de "back-office". En situación de servicio es necesario gestionar un sinnúmero de situaciones no previstas, por ello los agentes deben actuar ajustados a las características de las circunstancias. En este escenario las organizaciones deben facilitar o favorecer el uso de diversas y numerosas competencias, competencias que frecuentemente son subestimadas.

Se puede entonces identificar algunas de las competencias requeridas:

a. El agente debe desarrollar conocimientos técnicos y de relación personal para que la interacción con el beneficiario responda las necesidades específicas.

b. El colectivo de trabajo, debe poder acceder al conjunto de conocimientos y Now-how puesto a disposición por la organización.

c. El cliente debe reconocer el servicio como una interacción que existe y tiene sentido.

La relación de servicio es una relación que implica un número complejo, diversificado y creciente de interacciones con el cliente. Éstas se desarrollan en el marco de una transacción que está circunscrita por un número de reglas, procedimientos y medios fijados de antemano por la organización. En razón a la naturaleza cambiante de las solicitu- des (interrogantes sobre beneficios, opciones, cambios, ampliaciones del servicio, etc.) de los beneficiarios. De esta manera la relación de servicio se define a partir de las características específicas de cada una de las tareas a resolver.

En la prestación del servicio, la informatización juega un papel central, los cambios de herramientas y útiles de gestión de la información, a veces no están plenamente adaptados, esto ocasiona que no todas las solicitudes puedan ser atendidas y que el servicio pueda ser calificado de incompetente. Adicionalmente las prácticas de gestión prescriben ciertos comportamientos con un control frecuente sobre "las respuestas estandarizadas: $S B A G$ (sonreír, buenos días, adiós, gracias) o BOASGA (buen día, observar, atención, sonreír, gracias, adiós)".

El trabajo en las actividades de servicios, supone una colaboración mutua entre un prestatario y un beneficiario. La interacción que allí surge requiere la movilización elaborada de ciertos recursos técnicos de una de las partes (el prestatario). En esta interacción lo imprevisto se encuentra al orden del día, el desarrollo de un servicio de calidad depende de la posibilidad del prestatario de poder tomar la iniciativa en la co-realización de la actividad, por ello las contradicciones entre lo que se puede y no se puede hacer redunda en la satisfacción del cliente (desplazamiento o desvío de su solicitud).

Finalmente en las situaciones de trabajo que se definen por un servicio que consiste esencialmente en tratar informaciones de diversa naturaleza y de intercambiar conocimientos en el marco de interacciones complejas. En la unidad estudiada la relación de servicio supone relaciones de contacto, comunicaciones verbales (también no verbales) para recibir, escuchar, instruir, informar sobre hechos o eventos, argumentar, explicar, traducir, decir y contradecir.

En esta situación de servicio la relación está focalizada, se realiza cara a cara por los siguientes tipos de acciones: 
- Orientación: ayudar al beneficiario del servicio a ubicarse en el tiempo y en el espacio.

- Información: suministrar la información adecuada a las solicitudes del beneficiario.

- Reclamación: verificar y/o resolver los problemas de funcionamiento vinculados a la prestación del servicio.

- Asistencia: tratar las solicitudes del cliente personalizando las respuestas.

Desde el punto de vista de la metodología OCRA, el análisis depende de la identificación cuidadosa de los movimientos realizados implica una correcta diferenciación de los grados de libertad, de otro lado ciertos movimientos dependen de factores externos a la tarea, muchas veces de orden organizacional (número de clientes atendidos en este caso). Éstos deben identificarse para apreciar correctamente la continuidad, su frecuencia y presencia. Se debe igualmente diferenciar entre periodos de trabajo estabilizados (estándar) y periodos de trabajo con alteraciones derivadas de aspectos comerciales (competitividad), mercado (demandas de ciertos productos) planeación (uso de materiales y herramientas) organizacionales (contratación, temporalidad, etc.) o los relativos al mantenimiento de equipos y herramientas.

Por estas razones es esencial comprender que el método tiene como bondad la obtención de un índice que es el resultado de un proceso de observación y registro sistemático, en este caso el resultado es la presencia de riesgo medio; sin embargo, al exposición es relativa a la eficiencia en el desarrollo de la tarea por parte del agente y la relativa a los periodos de picos de demanda de los servicios. En este sentido, el método tiene limitantes en cuanto a su capacidad para diferenciar estrategias de operación de los trabajadores y ejecución automática de acciones. El método entonces ganará en precisión en la medida que se adopte una metodología participativa y se pueda integrar la experiencia del trabajador observado, también cuando se integran observaciones de diferentes trabajadores realizando las mismas tareas. Las implicaciones de estas consideraciones son relativas a las posibles modificaciones pero esencialmente al impacto y utilidad de las recomendaciones adoptadas.

Recibido: junio 2008

Aceptado: octubre 2008

\section{Bibliografía}

Bao S, Spielholz P, Howard N and Silverstein B. Quantifying repetitive hand activity for epidemiological research on musculoskeletal disorders - Part I: individual exposure assessment. Ergonomics, 2006; 49, 361-380.

Bystro' M, S.E.G. and Kilbom A. Physiological response in the forearm during and after isometric intermittent handgrip. European Journal of Applied Physiology, 1990; 60, 457-466.

Cook T, Rosencrance J, Zimmermann C, Gerleman $\mathrm{D}$ and Ludewig P. Electromyographic analysis of a repetitive hand gripping task. International Journal of Occupational Safety and Ergonomics, 1998; 4, 185-200.

Colombini D. An observational method for classifying exposure to repetitive movements of the upper limbs. Ergonomics, 1998; 41, 1261-1289.

Colombini D, Occhipinti E, Delleman N, Fallentin M, Kilbom A and Grieco A. Exposure assessment of upper limb repetitive movements: a Consensus Document. In International Encyclopedia of Ergonomics and Human Factors, W. Karwowski (ed.), (London: Taylor and Francis). 2001; 52-66.

Colombini D, Occhipinti E and Grieco A. Risk assessment and management of repetitive movements and exertions of upper limbs (Amsterdam: Elsevier Science). 2002.

Colombini D. and Occhipinti E. Risultati della valutazione del rischio e del danno in gruppi di lavoratori esposti, in diversi comparti lavorativi, a movimenti e sforzi ripetuti degli arti superiori. La Medicina del Lavoro, 2004; 95, 233-246. 
Dahalan JB and Fernandez J.E. Psychophysical frequency for a gripping task. International Journal of Industrial Ergonomics, 1993; 12, 219-230.

Jones LA and Hunter IW. Force and EMG correlates of constant effort contractions. European Journal of Applied Physiology, 1983; 51, 7583.

Hagberg M. Electromyography signs of shoulder muscular fatigue in two elevated arm positions. American Journal of Physical Medicine, 1981; 60, 111-121.

Mathiassen SE. the influence of exercise/rest schedule on the physiological and psychophysical response to isometric shoulder-neck exercise. European Journal of Applied Physiology, 1993; 67, 528-539.

Mathiassen SE and Winkel J. Quantifying variation in physical load using exposure - vs time data. Ergonomics, 1991; 12, 1455-1468.

Occhipinti E, OCRA: a concise index for the assessment of exposure to repetitive movements of the upper. Climbs. Ergonomics, 1998; 41, 1290-1311.

Occhipinti E. Colombini D, Cairoli S and Baracco A. Proposta e validazione preliminare di una checklist per la stima dell'esposizione lavorativa a movimenti e sforzi ripetuti degli arti superiori. La Medicina del Lavoro, 2000; 91, 470-485.

Roman-Liu D. Upper limb load as a function of repetitive task parameters. Part 1 - a model of the upper limb load. International Journal of Occupational Safety and Ergonomics, 2005; 11, 1-10.

Roman-Liu D, Tokarski T and Wo' Jcik K. Quantitative assessment of upper limb muscle fatigue depending on the conditions of repetitive task load. The Journal of Electromyography and Kinesiology, 2004; 14, 671-682.

Roman-Liu Kedzior K, Rzymkowski C. Computerized method for work space optimization in conditions of static work. International Journal of Occupational Safety and Ergonomics, 1999; 5 (1), 95-106.

West W, Hicks A, Clements L and Dowling J. The relationship between voluntary electromyogram, endurance time and intensity of effort in isometric handgrip exercise. European Journal of Applied Physiology, 1995; 71, 301-305. 